\title{
Severe Hypokalemia Secondary to Transient Distal Renal Tubular Acidosis in a Previously Healthy Woman
}

\author{
Efthymia Kallistrou $^{1}$, Nalini N. Architha ${ }^{2}$, Soubhik K. Pal ${ }^{3}$, Samson O. Oyibo ${ }^{4}$ \\ 1. General Medicine, Peterborough City Hospital, Peterborough, GBR 2. Emergency Medicine, Peterborough City \\ Hospital, Peterborough, GBR 3. Nephrology, Peterborough City Hospital, Peterborough, GBR 4. Diabetes and \\ Endocrinology, Peterborough City Hospital, Peterborough, GBR
}

Corresponding author: Efthymia Kallistrou, efthymia.kallistrou@nhs.net

\begin{abstract}
Normal anion gap (non-gap) hyperchloremic acidosis with hypokalemia is a medical emergency. There are several causes of this metabolic phenomenon, of which distal renal tubular acidosis is among the very rare causes. In this report, we present an unusual case of a previously healthy woman who was admitted to the intensive care unit with a short history of severe muscle weakness. She had no significant past medical history and was not taking any regular medication. There was also no history of recent drug or herb ingestion. Investigations demonstrated a combination of severe hypokalemia, hyperchloremia, hypobicarbonatemia (non-gap metabolic acidosis), and relatively raised urinary potassium and urinary $\mathrm{pH}$ in the presence of severe hypokalemia and metabolic acidosis. Results suggested a diagnosis of distal renal tubular acidosis. The patient responded rapidly to a short course of electrolyte replacement therapy and the condition resolved spontaneously thereafter. This case highlights the fact that distal renal tubular acidosis can occur as a transient phenomenon in previously healthy individuals.
\end{abstract}

Review began 01/17/2021 Review ended 01/17/2021 Published 01/18/2021

\section{() Copyright 2021}

Kallistrou et al. This is an open access article distributed under the terms of the Creative Commons Attribution License CC-BY 4.0., which permits unrestricted use, distribution, and reproduction in any medium, provided the original author and source are credited.
Categories: Emergency Medicine, Nephrology

Keywords: hypokalemia, transient, distal renal tubular acidosis, non-gap, hyperchloremia, hypobicarbonatemia, metabolic acidosis, urinary potassium, urinary ph, muscle weakness

\section{Introduction}

Distal renal tubular acidosis (dRTA) is a rare condition characterized by a failure to acidify the urine in the distal parts of the nephron, including the connecting tubule and the collecting duct. It is due to a transport defect involved in the secretion of hydrogen ions. The defect results in marked acid-base abnormalities, which include hyperchloremic metabolic acidosis and severe hypokalemia, which can be fatal [1,2]. This condition rarely occurs in adults due to infection, drugs, and autoimmune disorders [3]. It is commoner in children where it is usually due to a genetic defect in the acid-base regulatory system of the kidneys [4]. Cases of transient dRTA have been rarely reported in neonates in relation to other metabolic disorders but not in adults. We describe a case of a woman who presented to the emergency department with severe weakness due to severe hypokalemia, which was secondary to transient distal renal tubular acidosis.

\section{Case Presentation}

\section{Medical history and demographics}

A 66-year-old female presented to the emergency department with an inability to walk. She had noticed muscle ache and progressive weakness in both upper and lower limbs over the preceding two days. There was no associated history of vomiting, diarrhea, fever, seizures, paresthesia, or headache. No cough, breathlessness, or chest pain. There was no history of recent ingestion of any medication, herbal remedies, or recreational substances. Her past medical history included eczema and she was not on any regular medication. She was non-English speaking and had been living in the United Kingdom for the past nine years. She lived with her daughter at the time of the presentation. She reported smoking four cigarettes daily and denied alcohol intake. Clinical examination revealed a healthy-looking female, conscious and alert, with normal body temperature. She had bilateral upper limb weakness (power of $2 / 5$ ) and lower limb weakness (power of 3/5). There was no sensory deficit. Upper and lower limb muscle tone and reflexes, including plantar reflexes were normal. Other systems, including heart rate and respiratory rate, were normal. Blood pressure and oxygen saturation levels were also normal.

\section{Investigations}

The initial investigations revealed severe life-threatening hypokalemia, hyperchloremia, a low serum bicarbonate level, and a low blood $\mathrm{pH}$. A normal full blood count and C-reactive protein indicated the absence of any significant infection. A spot urine analysis showed a urinary potassium level higher than expected (>15 mmol/L) for the severe hypokalemia indicating excessive renal loss of potassium. The urinary $\mathrm{pH}$ was also higher than expected $(>5.5)$ for the metabolic acidosis indicating failure to acidify the urine at 


\section{Cureus}

the same time. Unfortunately, urine chloride and creatinine were not measured so we could not calculate the urinary anion gap or urinary potassium-to-creatinine ratio (Table 1 and Table 2). The patient's electrocardiogram demonstrated inverted T-waves, Q-T prolongation, prominent U-waves, and mild ST depression secondary to severe hypokalemia (Figure 1). An ultrasound scan demonstrated normal kidneys.

\begin{tabular}{|c|c|c|}
\hline Blood chemistry & Result & Reference range \\
\hline Sodium (mmol/L) & 146 & 132-145 \\
\hline Potassium (mmol/L) & 1.7 & 3.4-5.1 \\
\hline Chloride (mmol/L) & 120 & $97-110$ \\
\hline Bicarbonate (mmol/L) & 13 & $23-30$ \\
\hline Anion gap & 13 & 4-16 \\
\hline Creatinine (mmol/L) & 82 & $45-84$ \\
\hline Urea (mmol/L) & 5.8 & $2.5-7.8$ \\
\hline Serum corrected calcium (mmol/L) & 2.4 & $2.20-2.60$ \\
\hline Phosphate (mmol/L) & 0.83 & $0.8-1.5$ \\
\hline Magnesium (mmol/L) & 1.03 & $0.7-1.0$ \\
\hline Glucose (mmol/L) & 5.6 & 4.0-7.0 \\
\hline Thyroid stimulating hormone (mU/L) & 2.3 & $0.3-4.2$ \\
\hline 9-am cortisol (nmol/L) & 303 & $250-600$ \\
\hline Renin (mU) & 4.0 & $5.4-30$ \\
\hline Aldosterone (pmol/L) & $<70$ & $90-405$ \\
\hline Total protein (g/L) & 72 & $60-80$ \\
\hline Albumin (g/L) & 45 & $35-50$ \\
\hline Globulin (g/L) & 27 & $20-35$ \\
\hline Venous pH & 7.305 & 7.35-7.45 \\
\hline Venous $\mathrm{PCO}_{2}(\mathrm{kPa})$ & 4.59 & 4.27-6.40 \\
\hline Venous $\mathrm{PO}_{2}(\mathrm{kPa})$ & 3.10 & $4.27-6.40$ \\
\hline Venous bicarbonate (mmol/L) & 16.7 & $23-30$ \\
\hline Venous lactate (mmol/L) & 1.33 & $0.2-1.8$ \\
\hline
\end{tabular}

TABLE 1: Laboratory tests, including serum chemistry and venous blood gas analysis 


\section{Cureus}

\begin{tabular}{|c|c|c|}
\hline Spot urine test & Result & Expected range \\
\hline Sodium (mmol/L) & 85 & \\
\hline Potassium (mmol/L) & 17 & $<15.0$ \\
\hline Urea (mmol/L) & 115 & \\
\hline $\mathrm{pH}$ & 6.0 & $<5.5$ \\
\hline Specific gravity & 1.010 & $1.002-1.020$ \\
\hline
\end{tabular}

TABLE 2: Urine spot test

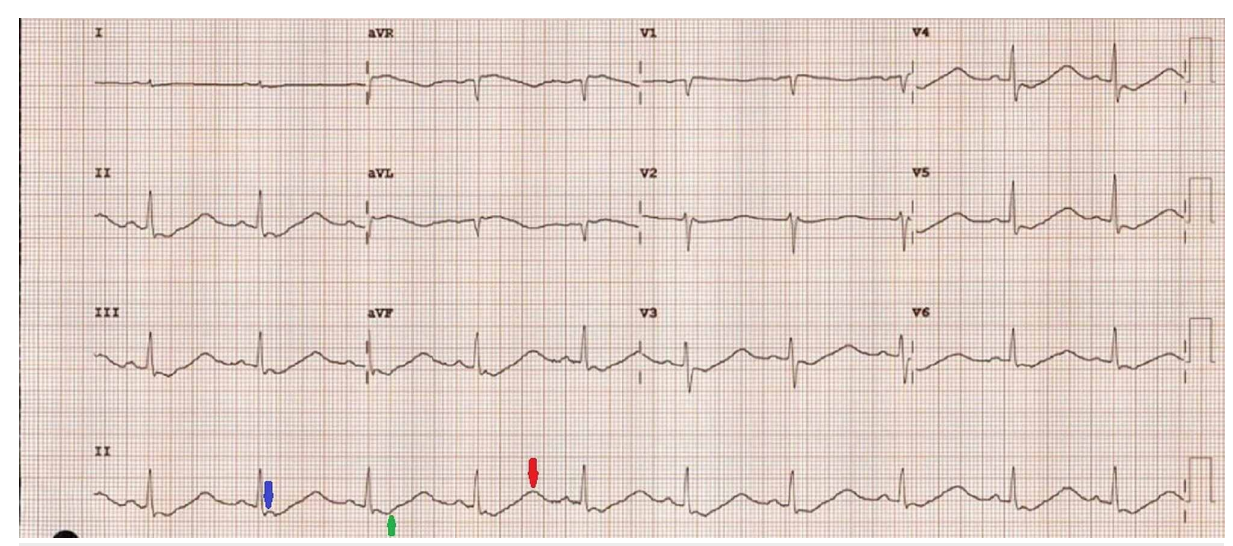

\section{FIGURE 1: Electrocardiogram performed during admission}

The initial electrocardiogram showing mild ST depression (blue arrow), inverted T-waves (green arrow), Q-T prolongation and prominent U-waves (red arrow) caused by severe hypokalemia.

Urine dipstick test for glucose, ketones, protein, and blood was negative. The coronavirus (COVID-19) screen was negative. The thyroid function, liver function, and overall renal function were normal. Reninaldosterone levels ruled out Conn's syndrome (hyperaldosteronism) as a cause of hypokalemia. The combination of hypokalemia, hyperchloremia, hypobicarbonatemia (non-gap hyperchloremic metabolic acidosis), and relatively raised urinary potassium and urinary $\mathrm{pH}$ in the presence of severe hypokalemia and metabolic acidosis, respectively, all suggested distal renal tubular acidosis.

\section{Treatment}

The patient was treated with intravenous potassium replacement and because of persistent hypokalemia and metabolic acidosis, the patient was transferred to the intensive care unit for replacement via a central venous line and closer monitoring. Her potassium levels normalized after 72 hours of treatment. Over three days of intensive therapy, she required a total of $516 \mathrm{mmol}$ of intravenous potassium and $72 \mathrm{mmol}$ of oral bicarbonate (as sodium bicarbonate). She was discharged shortly afterward with a short course of oral potassium ( $48 \mathrm{mmol} /$ day) and sodium bicarbonate ( $24 \mathrm{mmol} /$ day) to last for seven days.

\section{Outcome and follow-up}

The patient made a rapid and full recovery and was due to have a repeat serum potassium check a week after stopping the potassium and bicarbonate supplements. Further investigation results revealed normal serum immunoglobulin levels and normal autoimmune antibody screen ruling out autoimmune, autoinflammatory, and mixed connective tissue disorders (Table 3). The patient's serum potassium levels remained normal a week, a month, and three months after discharge and the patient remains well. 


\section{Cureus}

\begin{tabular}{|c|c|c|}
\hline Test & Result & $\begin{array}{l}\text { Reference } \\
\text { range }\end{array}$ \\
\hline Immunoglobulin G (g/L) & 9.6 & $6.0-16.0$ \\
\hline Immunoglobulin A (g/L) & 4.27 & $0.8-4.0$ \\
\hline Immunoglobulin M (g/L) & 0.39 & $0.5-2.0$ \\
\hline Anti-nuclear antibody (CU) & 4.3 & $<20.0$ \\
\hline Rheumatoid factor ( $\mathrm{mmol} / \mathrm{L}$ ) & $<12$ & $0.7-1.0$ \\
\hline Complement factor $3(\mathrm{~g} / \mathrm{L})$ & 0.87 & $0.75-1.65$ \\
\hline Complement factor $4(\mathrm{~g} / \mathrm{L})$ & 0.19 & $0.14-0.54$ \\
\hline $\begin{array}{l}\text { Other autoimmune antibodies including: Anti-double stranded DNA (anti-dsDNA), Anti-neutrophil } \\
\text { cytoplasmic antibody (ANCA), Anti-Sjögren's syndrome A (anti-SSA) (Ro60), Anti-Sjögren's syndrome B } \\
\text { (anti-SSB) (La), Anti-Jo-1, Anti-ribonucleic protein (anti-RNP), Anti-Smith (Anti-Sm), Anti-scleroderma } \\
\text { antibody (anti-Scl-70), Anti-ribosomal-P, Anti-centromere, Anti-Mi-2, Anti-Th/To, Anti-Ku, Anti-RNA } \\
\text { polymerase III, Anti-PM/Scl, Anti-proliferating cell nuclear antigen (anti-PCNA), Anti-liver (smooth muscle, } \\
\text { mitochondrial) antibodies, Anti-liver kidney microsomal type } 1 \text { antibodies (anti-LKM), Anti-glomerular } \\
\text { basement membrane antibody (anti-GBM) }\end{array}$ & Negative & \\
\hline Protein electrophoresis & $\begin{array}{l}\text { Normal, no } \\
\text { monoclonal } \\
\text { band seen }\end{array}$ & \\
\hline
\end{tabular}

TABLE 3: Protein electrophoresis and autoimmune workup

\section{Discussion}

Distal renal tubular acidosis (dRTA) is a condition that occurs as a result of impaired urinary acid excretion and inability to lower urinary $\mathrm{pH}$ despite the presence of acidosis. This is due to a defect in hydrogen ion secretion by the $\alpha$-intercalated cells in the distal convoluted tubules and the collecting tubules. Reduced excretion of ammonia ions in the urine is also present in affected individuals. Other significant sequelae of this condition include striking hypokalemia, hyperchloremic non-anion gap (non-gap) metabolic acidosis, hypocitraturia, hypercalciuria, and nephrocalcinosis [1-5]. Muscle weakness and muscle paralysis due to hypokalemia from renal potassium wastage is a prominent symptom [5]. Patients can also experience headaches, lack of energy, nausea, and vomiting as a result of acidosis. Depending on the severity of acidosis, stupor, coma, myocardial instability, or a cardiac arrest may occur. The body attempts to reduce carbon dioxide levels by a compensatory increase in respiratory rate however, in long-standing disease, this may lead to muscle fatigue and respiratory failure. In children, stunted growth and bone loss (rickets, osteomalacia) are often present because of chronic metabolic acidosis. The associated nephrocalcinosis, nephrolithiasis, and hypercalciuria can lead to the development of chronic kidney disease. Hearing loss is seen in distinct autosomal recessive types [5].

Distal renal tubular acidosis can be secondary due to systemic diseases or drug-induced or can be primary due to gene mutations. Several systemic or autoimmune have been associated with acquired dRTA. The underlying mechanism is believed to be related to direct renal tubular damage [5]. Table 4 shows a list of conditions associated with acquired (secondary) dRTA [1-5]. 


\section{Cureus}

Conditions associated with acquired distal renal tubular acidosis

Autoimmune conditions

Hypergammaglobulinemic states

Tubulointerstitial diseases

Genetic conditions

Drugs
Systemic lupus erythematosus (SLE), Sjögren's syndrome, rheumatoid arthritis, systemic sclerosis, thyroiditis, hepatitis, primary biliary cirrhosis

Monoclonal gammopathy, multiple myeloma, amyloidosis, cryoglobulinemia, chronic liver disease Chronic pyelonephritis, chronic interstitial nephritis, obstructive uropathy, renal transplant rejection Marfan's syndrome, Ehler-Danlos syndrome, sickle cell disease, congenital urinary tract obstruction Amphotericin B, lithium, non-steroidal anti-inflammatory drugs, toluene and pentamidine Familial hypercalciuria, chronic hypercalcemia, Wilson's disease

TABLE 4: Conditions associated with acquired distal renal tubular acidosis

Primary dRTA can occur sporadically or be inherited in an autosomal dominant or recessive pattern. So far, mutations have been identified in the following genes: the solute carrier family 4 member 1 (SLC4A1) gene that encodes a protein called anion exchanger 1 or AE1 that helps negatively-charged atoms cross cell membranes, the ATPase $\mathrm{H}+$ transporting V1 submit B1 (ATP6V1B1) gene and the ATPase $\mathrm{H}+$ transporting V0 submit A4 (ATP6VOA4) gene that encodes proteins that are part of a proton pump complex called vacuolar ATPase (V-ATPase) that helps positively-charged atoms cross cell membranes, the tryptophan-aspartate repeat domain 72 (WDR72) gene that encodes a protein possibly associated with intracellular endocytic vesicle trafficking, and the forkhead box i1 transcription factor (FOXI1) gene that encodes a protein involved in regulating the V-ATPase proton pump subunits in the inner ear, kidney, and epididymis [6,7].

The diagnosis of dRTA is based on a demonstration of the nephron's inability to lower urinary pH below 5.5 in the presence of systemic acidosis, normal anion gap (non-gap), hyperchloremia, and hypokalemia. Measurement of the urinary electrolytes should reveal a positive anion gap that reflects the decrease in urine ammonium excretion and can help differentiate between other causes of metabolic acidosis like diarrhea [5]. An electrocardiogram (ECG) should be performed in cases of moderate or severe hypokalemia to look for changes that can result in life-threatening dysrhythmias. Diagnostic work-up should always include the search for nephrocalcinosis by ultrasonography and the measurement of 24-hour urinary excretion of calcium, magnesium, and citrate. Furthermore, failure to lower urine $\mathrm{pH}$ below 5.3, either after ammonium chloride loading or after furosemide administration can establish the diagnosis of dRTA [1,3,8]. If no underlying cause is found then genetic testing will be required.

The treatment for dRTA in the acute setting consists of adequate potassium replacement and correction of the metabolic acidosis. Treatment of the underlying condition cannot be overemphasized. For chronic dRTA, oral bicarbonate replacement as sodium bicarbonate (1-2 mmol/ $\mathrm{kg} / \mathrm{day})$ or potassium citrate (1-2 $\mathrm{mmol} / \mathrm{kg} / \mathrm{day}$ ) is recommended until the plasma bicarbonate increases to at least $22 \mathrm{mmol} / \mathrm{L}$. Citrate salts also correct the hypocitraturia and prevent nephrolithiasis [7,9].

There are other causes of non-gap hyperchloremic metabolic acidosis with hypokalemia [10]. Non-gap metabolic acidosis can also occur with raised or normal serum potassium levels [10]. These causes are summarized in Table 5 below. 


\section{Cureus}

\section{Causes of non-gap metabolic acidosis}

Gastrointestinal Diarrhea, intestinal fistulae, laxative abuse, uretero-ileostomy, and uretero-sigmoidostomy

Conditions with Metabolic Ketoacidosis, lactic acidosis, and renal tubular acidosis

hypokalemia

Drugs

Administration of chloride-rich solutions and the use of acetazolamide in patients with renal dysfunction

Conditions with Administration of hydrogen and chloride ions in the form of total parenteral nutrition or ammonium chloride raised or normal administration, hyperkalemic dRTA due to hyporeninemic hypoaldosteronism, tubular resistance to aldosterone, serum aldosterone deficiency, chronic renal failure, Gordon's syndrome (pseudohypoaldosteronism), and administration

potassium levels of chloride-rich solutions

TABLE 5: Causes of normal anion gap (non-gap) metabolic acidosis

There are very few published case reports of transient dRTA. One such case described an elderly female who had transient dRTA after organophosphate poisoning. She had multiple ventricular arrhythmias due to hypokalemia but all resolved after treatment [11]. Another interesting report described a neonate with lifethreatening metabolic acidosis due to distal renal tubular acidosis and secondary hyperparathyroidism. This patient was diagnosed with Lightwood-Albright syndrome (transient infantile renal tubular acidosis) [12]. There is also a case report of a young child who had transient hyperkalemic dRTA with bicarbonate wasting with no underlying cause found [13].

We have presented a rare case of severe hypokalemia secondary to transient distal RTA in a previously healthy woman. There was no underlying cause identified for this patient's presentation, the patient responded rapidly to a short course of electrolyte replacement therapy and the condition resolved spontaneously after a few days.

\section{Conclusions}

Severe life-threatening hypokalemia and hyperchloremic acidosis due to distal RTA can occur as a transient phenomenon. There are other more common causes of non-gap hyperchloremic acidosis that can easily be excluded by adhering to a systematic approach. It is possible that our patient had an underlying cause for transient dRTA; however, despite extensive investigation, no cause was identified. We hope that this case report will not only add to the existing literature but also contribute to the heightened awareness of transient distal renal tubular acidosis.

\section{Additional Information}

\section{Disclosures}

Human subjects: Consent was obtained by all participants in this study. Conflicts of interest: In compliance with the ICMJE uniform disclosure form, all authors declare the following: Payment/services info: All authors have declared that no financial support was received from any organization for the submitted work. Financial relationships: All authors have declared that they have no financial relationships at present or within the previous three years with any organizations that might have an interest in the submitted work. Other relationships: All authors have declared that there are no other relationships or activities that could appear to have influenced the submitted work.

\section{References}

1. Soriano JR: Renal tubular acidosis: the clinical entity . J Am Nephrol. 2002, 13:2160-2170 10.1097/01.ASN.0000023430.92674.E5

2. Bagga A, Sinha A: Renal tubular acidosis. Indian J Pediatr. 2020, 87:733-744. 10.1007/s12098-020-03318-8

3. Both T, Zietse R, Hoorn EJ, et al.: Everything you need to know about distal renal tubular acidosis in autoimmune disease. Rheumatol Int. 2014, 34:1037-1045. 10.1007/s00296-014-2993-3

4. Besouw MTP, Bienias M, Walsh P, et al.: Clinical and molecular aspects of distal renal tubular acidosis in children. Pediatr Nephrol. 2017, 32:987-996. 10.1007/s00467-016-3573-4

5. Vallés PG, Batlle D: Hypokalemic distal renal tubular acidosis. Adv Chronic Kidney Dis. 2018, 25:303-320. 10.1053/j.ackd.2018.05.003

6. Alexander RT, Law L, Gil-Peña H, Greenbaum LA, Santos F: Hereditary distal renal tubular acidosis . GeneReviews ${ }^{\circledR}$. Adam MP, Ardinger HH, Pagon RA (ed): University of Washington, Seattle, WA; 2019.

7. Watanabe T: Improving outcomes for patients with distal renal tubular acidosis: recent advances and challenges ahead. Pediatric Health Med Ther. 2018, 9:181-190. 10.2147/PHMT.S174459

8. Walsh SB, Shirley DG, Wrong OM, Unwin RJ: Urinary acidification assessed by simultaneous furosemide and fludrocortisone treatment: an alternative to ammonium chloride. Kidney Int. 2007, 71:1310-1316. 10.1038/sj.ki.5002220 


\section{Cureus}

9. Domrongkitchaiporn S, Khositseth S, Stitchantrakul W, et al.: Dosage of potassium citrate in the correction of urinary abnormalities in pediatric distal renal tubular acidosis patients. Am J Kidney Dis. 2002, 39:383391. 10.1053/ajkd.2002.30560

10. Kraut JA, Madias NE: Differential diagnosis of nongap metabolic acidosis: value of a systematic approach . Clin J Am Soc Nephrol. 2012, 7:671-679. 10.2215/CJN.09450911

11. Narayan R, Abdulla MC, Alungal J: Transient distal renal tubular acidosis in organophosphate poisoning . Indian J Crit Care Med. 2017, 21:170-171. 10.4103/ijccm.IJCCM_298_16

12. Igarashi T, Sekine Y, Kawato H, Kamoshita S, Saigusa Y: Transient neonatal distal renal tubular acidosis with secondary hyperparathyroidism. Pediatr Nephrol. 1992, 6:267-269. 10.1007/BF00878365

13. Khositseth S: Transient hyperkalemic distal renal tubular acidosis with bicarbonate wasting in a young child. J Med Assoc Thai. 2011, 94:204-207. 